\title{
The CT20 peptide causes detachment and death of metastatic breast cancer cells by promoting mitochondrial aggregation and cytoskeletal disruption
}

\author{
MW Lee ${ }^{1,5}$, R Bassiouni ${ }^{2,5}$, NA Sparrow ${ }^{2}$, A lketani ${ }^{2}$, RJ Boohaker ${ }^{2}$, C Moskowitz ${ }^{1}$, P Vishnubhotla ${ }^{3}$, AS Khaled ${ }^{3}$, J Oyer ${ }^{2}$, A Copik $^{2}$, \\ C Fernandez-Valle ${ }^{2}$, JM Perez and AR Khaled ${ }^{4,2}$
}

Metastasis accounts for most deaths from breast cancer, driving the need for new therapeutics that can impede disease progression. Rationally designed peptides that take advantage of cancer-specific differences in cellular physiology are an emerging technology that offer promise as a treatment for metastatic breast cancer. We developed CT20p, a hydrophobic peptide based on the $C$ terminus of Bax that exhibits similarities with antimicrobial peptides, and previously reported that CT20p has unique cytotoxic actions independent of full-length Bax. In this study, we identified the intracellular actions of CT20p which precede cancer cell-specific detachment and death. Previously, we found that CT20p migrated in the heavy membrane fractions of cancer cell lysates. Here, using MDA-MB-231 breast cancer cells, we demonstrated that CT20p localizes to the mitochondria, leading to fusion-like aggregation and mitochondrial membrane hyperpolarization. As a result, the distribution and movement of mitochondria in CT20p-treated MDA-MB-231 cells was markedly impaired, particularly in cell protrusions. In contrast, CT20p did not associate with the mitochondria of normal breast epithelial MCF-10A cells, causing little change in the mitochondrial membrane potential, morphology or localization. In MDA-MB-231 cells, CT20p triggered cell detachment that was preceded by decreased levels of $\alpha 5 \beta 1$ integrins and reduced F-actin polymerization. Using folate-targeted nanoparticles to encapsulate and deliver CT20p to murine tumors, we achieved significant tumor regression within days of peptide treatment. These results suggest that СT20p has application in the treatment of metastatic disease as a cancer-specific therapeutic peptide that perturbs mitochondrial morphology and movement ultimately culminating in disruption of the actin cytoskeleton, cell detachment, and loss of cell viability.

Cell Death and Disease (2014) 5, e1249; doi:10.1038/cddis.2014.225; published online 22 May 2014

Subject Category: Experimental Medicine

Mitochondria are promising therapeutic targets given their role in regulating metabolism, cell death, and powering cytoskeletal changes involved in cell motility. ${ }^{1-4}$ Emerging evidence in the literature indicates that the ability of breast cancer cells to migrate and metastasize, which depends on cytoskeletal changes, may be linked to mitochondrial morphology and subcellular localization. ${ }^{5,6}$ Thus disrupting mitochondrial function would not only impact energy production but also impact cell adhesion and migration. What is lacking are new therapeutic agents that modulate mitochondrial dynamics and decrease the ability of this organelle to move and produce localized amounts of ATP that drive cytoskeletal change.
Such an agent could effectively impair cancer cell invasion, attenuating migration, and inducing the death of highly metastatic cancer cells.

Therapeutic peptides that can target the mitochondria of cancer cells and induce cell death hold significant promise and may represent an avenue to restrain migration and metastasis. ${ }^{7}$ Many cancer therapeutic peptides fall under the umbrella of naturally occurring antimicrobial peptides that form pores in membranes, facilitating the release of intramembrane contents. ${ }^{7,8}$ In mammalian systems, these peptides are relatively inactive against the plasma membrane, but if transfected or delivered into the cell, they exhibit

\footnotetext{
${ }^{1}$ Department of Medical Education, College of Medicine, University of Central Florida, Orlando, FL, USA; ${ }^{2}$ Burnett School of Biomedical Science, College of Medicine, University of Central Florida, Orlando, FL, USA; ${ }^{3}$ Orlando Veteran's Administration Medical Center, University of Central Florida, Orlando, FL, USA and ${ }^{4}$ Nanoscience Technology Center, University of Central Florida, Orlando, FL, USA

*Corresponding author: AR Khaled, Burnett School of Biomedical Sciences, College of Medicine, University of Central Florida, 6900 Lake Nona Blvd, Orlando, FL 32827 , USA. Tel: +1 850407266 7035; Fax: +1 850407266 7003; E-mail: annette.khaled@ucf.edu

${ }^{5}$ These authors contributed equally to this work.

Keywords: metabolism; cancer therapy; fission/fusion; actin; nanoparticles

Abbreviations: $\Delta \psi$, mitochondrial membrane potential; ATP, adenosine triphosphate; $\mathrm{COOH}$, carboxylated nanoparticles; CT20p, CT20 peptide encapsulated in nanoparticles; CTRL, control conditions; DAPI, fluorescent nuclear stain; DIC, differential interference contrast microscopy; DOX, doxorubicin encapsulated in nanoparticles; DMEM, dulbecco's modified Eagle's Media; DMSO, dimethyl sulfoxide; Drp1, dynamin related protein 1; ER, endoplasmic reticulum; FCCP, carbonyl cyanide-p-trifluoromethoxyphenylhydrazone; FOL, folate-decorated nanoparticles; H \& E, hemotoxylin and eosin; HBPE-NPs, hyperbranched polyester nanoparticles; JC-1, fluorescent mitochondrial stain and indicator of mitochondrial membrane potential; MCF-10A, non-cancerous breast epithelial cell line; MDA-MB-231, cancerous breast epithelial cell line; Mdivi-1, small molecule inhibitor of Drp1; MFN2, mitofusin 2; MFN2-YFP, YFP labeled mitofusin 2; NA, numerical aperture; OPA1, optic atrophy 1; PC, Pearson's coefficient of correlation for colocalization studies; RHO-CT20p, rhodamine-labeled CT20p; UNT, untreated control; VDAC-1, voltage-dependent anion channel 1
}

Received 29.1.14; revised 15.4.14; accepted 18.4.14; Edited by M Agostini 
pore-forming activity towards the prokaryotic-like membrane of the mitochondria. Considerable effort has been expended in developing synthetic peptides to trigger mitochondrial membrane permeability changes and promote cytochrome $C$ release and apoptosis; however, these approaches do not specifically harness differences between normal and cancerous cells, which often harbor mutations in proteins that govern apoptosis. An attractive alternative is to develop peptides from endogenous mitochondrial-localized proteins with documented functions. Numerous examples of this strategy exist, including the Nur77 peptide, VDAC-1 peptides, and more recently peptides derived from Bax. ${ }^{9-13}$ Despite the advantages of these approaches, many concerns remain, such as bioavailability and off-target effects.

We previously reported that a peptide derived from the $\mathrm{C}$ terminus of Bax, CT20p, had similarities to antimicrobial peptides, ${ }^{11}$ forming pores in artificial lipid vesicles and releasing intravesicular contents. ${ }^{14,15}$ When expressed in cells, CT20p localized to the mitochondria, promoting cell death, even in cells deficient in Bax, indicating that the peptide functioned independently of the parent protein and potential defects in the apoptotic machinery. ${ }^{11}$ However, the hydrophobic nature of CT20p, its inability to penetrate plasma cell membranes, and general problems with peptide stability in serum challenged the direct use of CT20p. An optimal solution was encapsulation of CT20p in hyperbranched polymeric nanoparticles (HBPE-NPs) that could be decorated with targeting ligands to concentrate the peptide in tumors. ${ }^{11,16}$ CT20p-HBPE-NPs (henceforth referred to as CT20p) killed colon and breast cancer cells, causing tumor regression in mice. ${ }^{11}$ Nevertheless, the key intracellular actions of CT20p that led to cell death remained unknown. Herein we report that CT20p impairs mitochondrial movement and distribution and has deleterious effects on integrins and F-actin polymerization, causing cell detachment and death in a cancer-specific manner. These findings suggest that CT20p could have potential clinical use as a mitochondrial-targeting, antimetastatic agent.

\section{Results}

CT20p localizes to mitochondria in breast cancer cells and causes cell death. As we previously reported, treatment conditions and cell viability are routinely assessed with each batch of nanoparticles, loaded with CT20p, by measuring percent death (indicated by increased membrane asymmetry), to establish the concentration that kills $\sim 50 \%$ of cancer cells in $48 \mathrm{~h}^{11}$ This is $\sim 3.5-4.0 \mathrm{nM}$ CT20p or 75-100 $\mu \mathrm{g}$ nanoparticles/ml (Supplementary Figures $1 \mathrm{~A}$ and B), which was the dose used for all experiments performed. In Figure 1a, using the metastatic breast cancer cell line, MDAMB-231, and the breast epithelial cell line, MCF-10A, a timecourse assessment of death after CT20p treatment is shown. No markers of late apoptotic or necrotic cell death were detected at 3 or $6 \mathrm{~h}$ post CT20p treatment in MDA-MB-231 or MCF-10A cells. We then confirmed our previous findings that death of MDA-MB-231 cells, indicated by changes in plasma membrane symmetry and permeability, was detectable 24-48 h after CT20p treatment. In contrast, MCF-10A cells were more resistant to the cytotoxic effects of CT20p at all the time points examined (Figure 1a). Hence, the intramolecular events that underlie the cancer-specific cell death induced by CT20p likely occur within the first few hours of treatment before cells display characteristic features of cell death. The cytotoxic effects of CT20p were not cell line specific because we observed a similar cancer-specific killing trend using freshly isolated breast tumor cells and normal breast epithelial cells from a breast cancer patient. In Figure $1 \mathrm{~b}$, we show that CT20p killed $\sim 47 \%$ of the breast tumor cells by $48 \mathrm{~h}$, but $<10 \%$ of the normal breast epithelial cells.

To track the intracellular localization of CT20p, we labeled the $\mathrm{N}$ terminus of the peptide with rhodamine $(\mathrm{RHO})$ and encapsulated RHO-CT20p in HBPE-NPs. Previously, we detected CT20p in mitochondrial-enriched cell fractions. ${ }^{11}$ To examine the association of CT20p with intracellular organelles, we stained cells with Mitotracker Green or ER-Tracker Green. Live cell confocal microscopy enabled the detection of colocalized RHO-CT20p (red fluorescence) with organelles (green fluorescence). In MDA-MB-231 and MCF-10A cells, internalization of RHO-CT20p was observed within 1 hour, and visualization of total red fluorescence indicated that uptake was equivalent (Figure $2 \mathrm{a}$ and Supplementary Figure 2). Colocalization of RHO-CT20p with mitochondria was detected between $1-3 \mathrm{~h}$ in MDA-MB-231 cells but not in MCF-10A cells (Figure 2a). CT20p did not colocalize with the endoplasmic reticulum in either cell type (Figure 2b and Supplementary Figure 2B). Quantitation (Pearson's coefficient (PC)) of the colocalization of CT20p with organelles was based on correlating the strength of the linear relationship between the red and green fluorescent channels. CT20p associated with mitochondria in MDA-MB-231 cells $(P C=0.909)$ but less so in MCF-10A cells $(P C=0.136)$ (Figure $2 c)$. Z-planes from the composite images in Figure 2 are shown in Supplementary data (Supplementary Figures $2 \mathrm{~A}$ and $\mathrm{B}$ ). These results suggest that the initial association of CT20p with mitochondria may depend on inherent metabolic or morphological differences that are unique to breast cancer cells.

To examine changes in mitochondrial morphology and $\Delta \psi$ as a consequence of CT20p treatment, we used the mitochondrial-specific dye, JC-1. The monomeric form of JC-1 in the cytosol fluoresces green, whereas mitochondrialassociated JC-1 fluoresces red, with its intensity dependent on the $\Delta \psi$. MDA-MB-231 and MCF-10A cells, treated with CT20p for 0 and $3 \mathrm{~h}$, were stained with $\mathrm{JC}-1$, and fluorescence was analyzed by flow cytometry. At the $3 \mathrm{~h}$ time point, MDA-MB-231 cells, in contrast to MCF-10A cells, exhibited marked $\Delta \psi$ hyperpolarization upon CT20p treatment (Figure 2d). These results were confirmed by confocal microscopy (Figure 2e). Moreover, CT20p altered the distribution of mitochondria in MDA-MB-231 cells but not in MCF-10A cells, causing significant clustering of mitochondria (Figure 2e).

CT20p promotes the fusion-like aggregation of mitochondria. To investigate the nature of the mitochondrial aggregation observed in CT20p-treated MDA-MB-231 cells (Figure 2e), we next examined mitochondrial fusion, using cells transfected with mitoDendra, and time-lapse, laser 
a

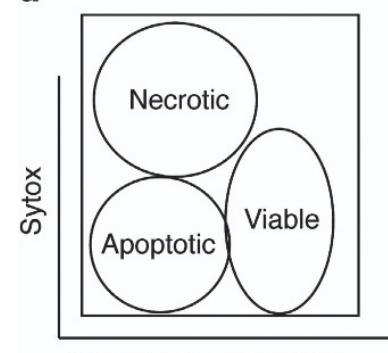

Membrane Asymmetry
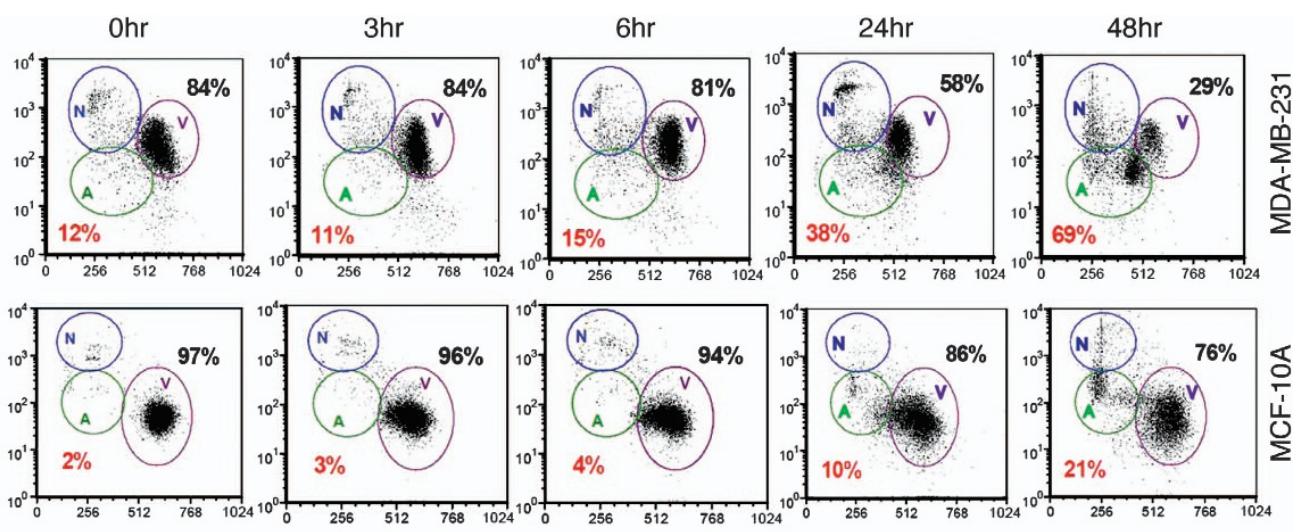

b Normal Breast Epithelium
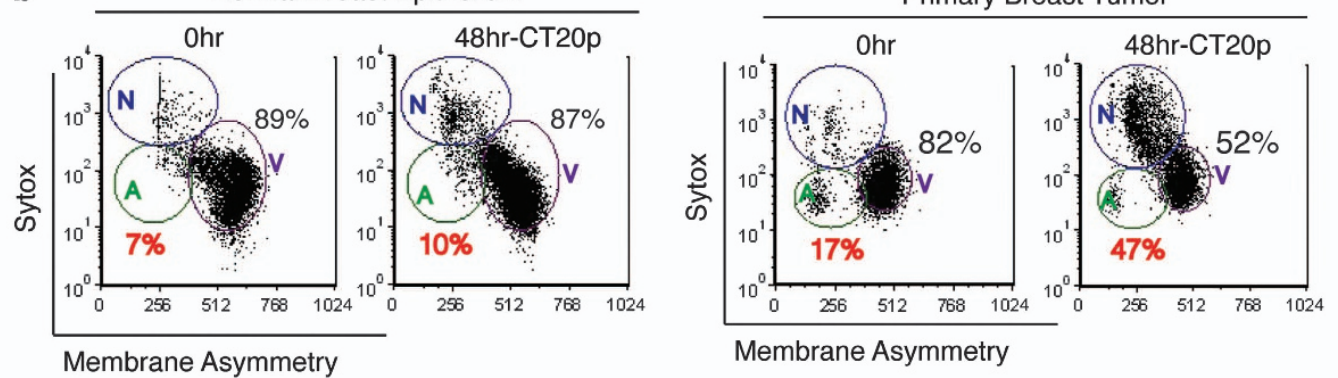

Figure 1 CT20p causes breast cancer cell-specific death. (a) MDA-MB-231 cells and MCF-10A cells were treated with CT20p, delivered in nanoparticles as described in Materials and Methods, for the indicated time points. Cells were stained with Sytox AADvanced and F2N12S dyes, and cell viability was assessed by flow cytometry. Three cell populations are identified and denoted as V (viable), A (apoptotic), and N (necrotic). Percentages of viable cells (black) and combined apoptotic/necrotic cells (red) are shown. (b) Normal breast epithelial cells and primary breast tumor cells were isolated as described in the Materials and Methods, treated with CT20p and cell viability examined as described above

scanning confocal microscopy was performed. ${ }^{17}$ Photoconversion of mitoDendra in a restricted region of the cell generated red mitochondria that were monitored over time for fusion with unconverted green mitochondria. In Figure $3 \mathrm{a}$, a schematic shows the experimental design. Images were captured at $120 \mathrm{~min}$ and $180 \mathrm{~min}$, encompassing a CT20p treatment window from $1-1 / 2$ to $3 \mathrm{~h}$. As shown in Figure $3 \mathrm{~b}$, mitochondria in control MDA-MB-231 cells did not fuse in the time interval observed. Note that in the DIC image, green fluorescent mitochondria in control MDA-MB-231 cells were distributed throughout the cell, especially at the extensions or protrusions (Figure 3b), and cell movement (see arrows) was captured, highlighting the heightened motility of these cells. In MDA-MB-231 cells treated with CT20p, fusion of red and green mitochondria was visible as indicated by the appearance of yellow fluorescence (Figure 3c). The DIC images of CT20p-treated MDA-MB-231 cells revealed the aggregation of green fluorescent mitochondria (Figure $3 c$ ) that was previously observed upon JC-1 staining (Figure 2e). In control or CT20p-treated MCF-10A cells, which are flatter cells, no mitochondrial fusion was detected (Figures $3 \mathrm{~d}$ and e). DIC images verify cell attachment throughout imaging. Additional controls, not shown, included the use of an irrelevant peptide that resulted in outcomes similar to the untreated controls.

To determine whether CT20p affected mitochondrial fusion proteins, the mitochondrial localization of mitofusin 2 (MFN2) $^{18,19}$ and optic atrophy 1 (OPA1) ${ }^{20}$ were examined by immunoblot (Figures $3 f$ and g). In MDA-MB-231 cells, MFN2 and OPA1 increased following $3 \mathrm{~h}$ of treatment with CT20p (Figure 3f) and coincided with the time points at which mitochondrial fusion was observed (Figure 3c). In contrast, mitochondrial-localized MFN2 was undetectable in CT20ptreated or untreated MCF-10A cells, and only a slight increase in OPA1 occurred (Figure 3g). Previously, others had shown that overexpression of MFN2 caused mitochondrial clustering and cell death. ${ }^{21}$ To determine whether overexpressing MFN2 could mimic the effects of CT20p, we transiently transfected cells with MFN2-YFP. Cells were also stained with Mitotracker Red. Overexpression of MFN2-YFP in MDA-MB-231 and MCF-10A cells caused membrane blebbing and morphological changes associated with cell death (Supplementary Figure $3 \mathrm{~A}$ and Supplementary movies). Moreover, blocking fission with Mdivi- $1,{ }^{22}$ a small molecule inhibitor of the fission protein Drp1, in MDA-MB-231 cells, caused similar effects on mitochondria clustering, followed by mitochondrial depolarization and cell death (Supplementary Figure 3B). Based on these results, perturbing the mitochondrial fission/fusion machinery in cancer cells may represent a viable means for triggering cell death that could, in part, account, for the actions of CT20p.

CT20p impairs mitochondrial distribution. Referring to the DIC images in Figure 3c, CT20p-treated MDA-MB-231 cells exhibited a rounded morphology with decreased membrane protrusions. Closer examination of mitochondrial 

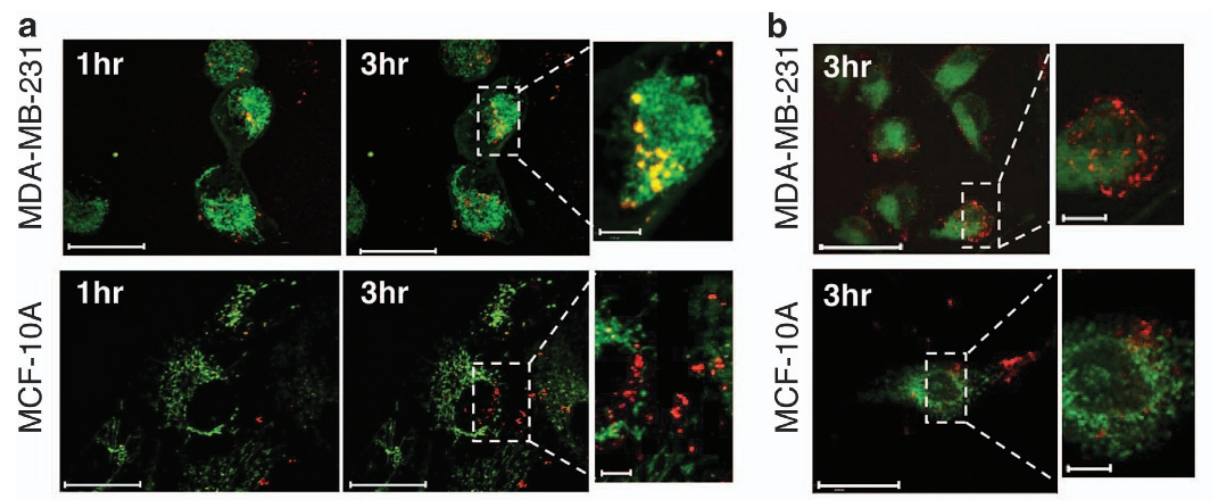

Rho-CT20p/Mitotracker Green

Rho-CT20p/ERtracker Green

d

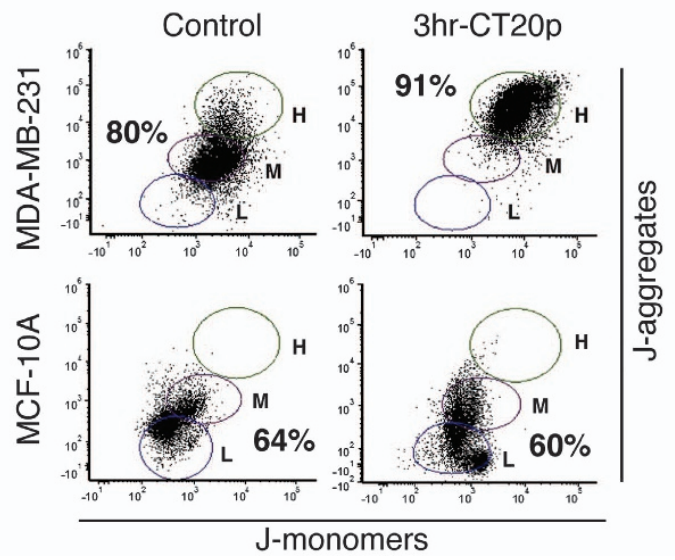

e
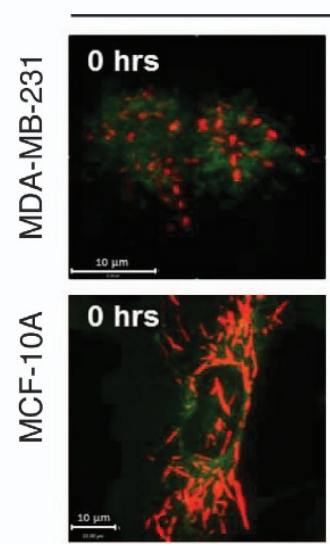

c

\begin{tabular}{|l|c|}
\hline & $\begin{array}{c}\text { Co-localization } \\
\text { Coefficient }\end{array}$ \\
\hline $\begin{array}{l}\text { MDA-MB-231 } \\
\text { CT20p \& Mito }\end{array}$ & $0.909 \pm 0.052^{\star}$ \\
\hline $\begin{array}{l}\text { MCF-10A } \\
\text { CT20p \& Mito }\end{array}$ & $0.136 \pm 0.067^{\star}$ \\
\hline $\begin{array}{l}\text { MDA-MB-231 } \\
\text { CT20p \& ER }\end{array}$ & $0.006 \pm 0.004$ \\
\hline $\begin{array}{l}\text { MCF-10A } \\
\text { CT20p \& ER }\end{array}$ & $0.007 \pm 0.006$ \\
\hline
\end{tabular}

JC-1
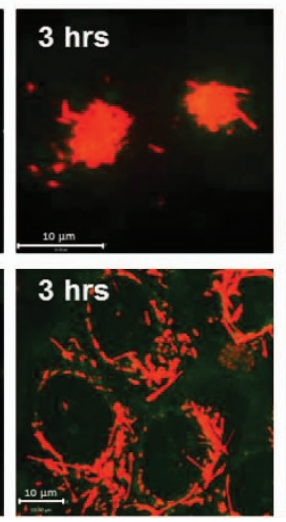
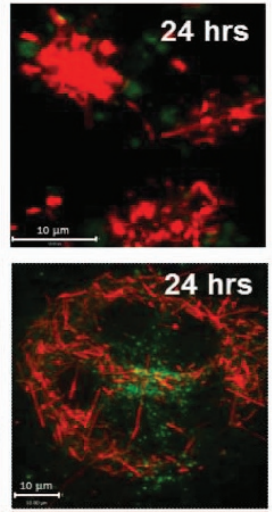

Figure 2 CT20p localizes to the mitochondria and increases the mitochondrial membrane potential in breast cancer cells. (a, b) MDA-MB-231 and MCF-10A cells were treated with RHO-labeled CT20p (RHO-CT20p), delivered in nanoparticles as described in Materials and Methods, stained with Mitotracker green (a) or ER-tracker green (b) for the indicated times, and analyzed by live cell imaging. Colocalization is denoted by yellow fluorescence. Scale shown is $25 \mu \mathrm{M}$ (MDA-MB-231) and 10 $\mu \mathrm{M}$ (MCF-10A). Inset magnification scale is $5 \mu \mathrm{M}$. (c) Colocalization coefficients of RHO-CT20p were calculated from the data in (a) and (b). ${ }^{*} P>0.05$. (d, e) MDA-MB-231 and MCF-10A cells were treated with CT20p as in (a, b), stained with the mitochondrial permeable dye, JC-1, and analyzed by flow cytometry (d) and confocal microscopy (e). H, high; M, mid; and L, low indicate mitochondrial membrane potential. Scale shown is $10 \mu \mathrm{M}$

distribution in CT20p-treated MDA-MB-231 cells by confocal microscopy at $2 \mathrm{~h}$ post treatment revealed the retraction of mitochondria (stained by MitoTracker Green) from membrane ruffles/protrusions (Figure 4a). In contrast, mitochondria in MCF-10A cells were localized throughout the cell, even after CT20p treatment (Figure 4b). A possible explanation for the altered mitochondrial distribution in CT20p-treated cells is that the peptide was impairing mitochondrial movement. To examine this, cells treated with CT20p were stained with MitoTracker Green and time-lapse images of mitochondrial movement were obtained. The velocity of mitochondrial movement was determined by tracking individual mitochondria using post-analysis software. This analysis indicated that the mitochondria of MDA-MB-231 cells were moving faster than the mitochondria of MCF-10A cells, and that mitochondrial movement was reduced by CT20p treatment (Figure 4c: see arrows). Calculation of average mitochondrial velocities under each condition revealed that CT20p caused a fourfold decrease in mitochondrial velocity in MDA-MB-231 cells as compared with control cells (Figure $4 d$ ).

As CT20p treatment significantly impacted mitochondrial movement, we next examined mitochondrial bioenergetics.
Cellular ATP levels were measured $3 \mathrm{~h}$ post CT20p treatment and a decrease upon CT20p treatment in MDA-MB-231 cells but not MCF-10A cells was noted in comparison with untreated cells or control nanoparticles (Figures $4 e$ and f). Next, we examined the effect of CT20p upon oxygen consumption and mitochondrial coupling efficiency at the time points that we observed mitochondrial clustering (3-6 h post СT20p treatment). Whereas we observed inherent differences in mitochondrial respiration and coupling efficiency between MDA-MB-231 and MCF-10A cells, CT20p had little to no effect on these processes (Figure $4 \mathrm{~g}$ ). As the mitochondrial respiration machinery seemed unperturbed by CT20p at the time points examined, localized loss of ATP owing to mitochondrial aggregation or extracellular release could account for decreased ATP levels (Figure 4e). As it was possible that mitochondrial fusion could alter cytoskeletal dynamics, ${ }^{6}$ we then sought to determine if the action of CT2Op upon mitochondria could lead to loss of cell adhesion and deregulate the cytoskeleton.

CT20p disrupts cell attachment and cytoskeletal organization. Localization of mitochondria to cell protrusions is critical for cancer cell migration. ${ }^{6,23}$ Loss of mitochondria 


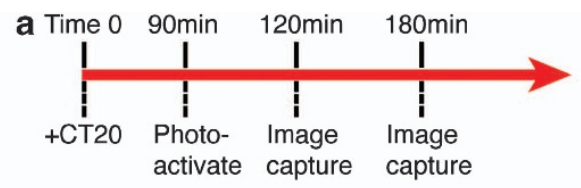

b

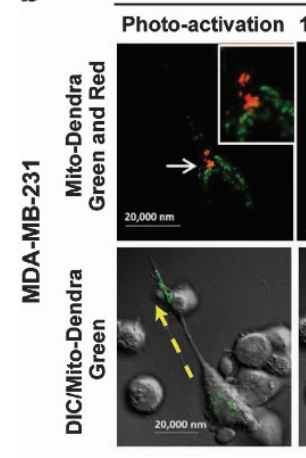

CTRL

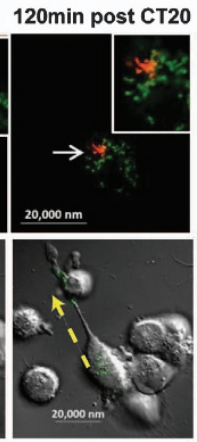

C

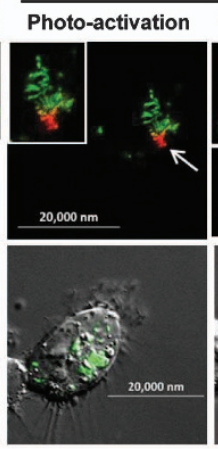

CT20p

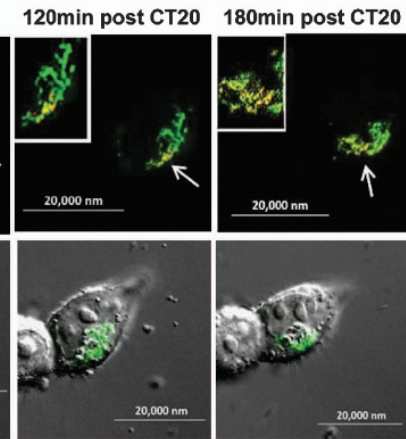

CT20p

d

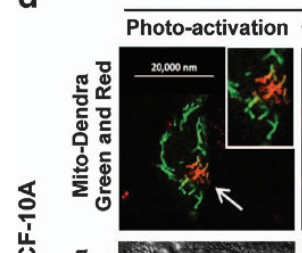

CTRL

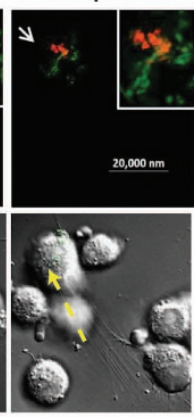

e
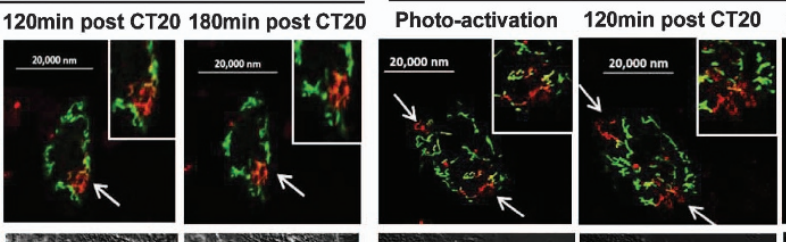

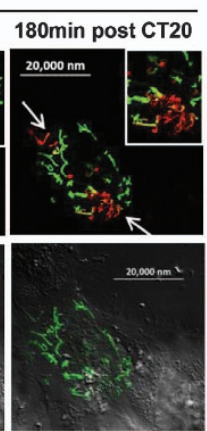

f

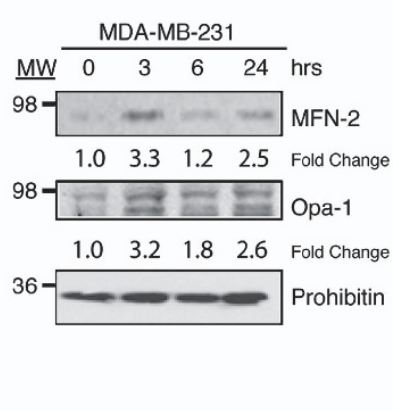

g

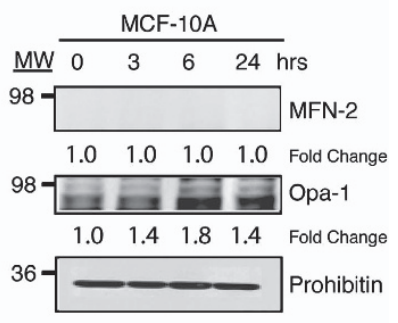

Figure 3 CT20p causes fusion of mitochondria in breast cancer cells. (a) Experimental plan is shown in schematic. At time 0, cells expressing MitoDendra, a photoactivatable mitochondrial targeted fluorescent protein to monitor fusion, were treated with CT20p, delivered in nanoparticles, as described in Materials and Methods. After $90 \mathrm{~min}$, cells were photo-activated to produce red mitochondria and images were acquired 30 (120 min post CT20p treatment) and 60 min later (180 min post CT20p treatment). Mitochondrial fusion was thus observed between 1-1/2 to $3 \mathrm{~h}$ post CT20p treatment. (b-e) MDA-MB-231 cells (b, c) or MCF-10A cells (d, e) were treated as described in (a). The green represents unconverted mitoDendra, the red is photo-activated mitoDendra, and the yellow results from fusion of red and green mitochondria. Arrows and inset indicate areas of photo-activation and movement of cells. Cells were untreated (CTRL) (b, d) or treated with CT20p (c, e) as described in (a) Representative images were acquired from time-lapse movies taken over a 90-minute period. Scale shown is $20,000 \mathrm{nM}$ or $20 \mu \mathrm{M}$. The scaling of the images was adjusted in panel $\mathbf{b}$ to show the movement of cells within a larger field of vision. Similar movement was not observed in MCF-10A cells or in MDA-MB-231 cells upon treatment with CT20p. (f, $\mathbf{g}$ ) Mitochondria lysates from MDA-MB-231 cells (f) and MCF-10A cells (g), treated with CT20p delivered in nanoparticles, were subjected to SDS-PAGE and immunoblotted for fusion proteins, MFN2 and OPA1. Prohibitin is a loading control for mitochondrial proteins. Fold changes were determined by densitometry for each representative blot. Images were cropped to improve presentation

redistribution to these regions following CT20p treatment could have detrimental consequences on cell attachment, which we examined using a crystal violet-based cellular adhesion assay. Briefly, cells were seeded to dishes coated with fibronectin, followed by treatment with CT20p. ${ }^{20}$ In contrast to the CT20p-treated MCF-10A cells that remained attached, MDA-MB-231 cells detached from the substrate by $6 \mathrm{~h}$ of CT20p treatment (Figures $5 \mathrm{a}$ and b). Subsequent iterations of this experiment with either uncoated plates or control nanoparticles revealed similar results. Based on these observations, we next determined whether CT20pmediated detachment of MDA-MB-231 cells was accompanied by alterations in integrins that are aberrantly expressed in breast cancer. ${ }^{24-27}$ Cells were treated with CT20p, and $\alpha 5$ and $\alpha \mathrm{V}$ integrins were detected by immunoblot (Figure $5 \mathrm{c}$ ), whereas $\beta 1$ and $\beta 3$ integrins were assessed by flow cytometry (Figure $5 \mathrm{~d}$ ). In MDA-MB-231, but not MCF-10A cells, $\beta 1$ decreased after $3 \mathrm{~h}$ of CT20p treatment, whereas surface levels of $\beta 3$ were not altered until after $24 \mathrm{~h}$ (Figure 5d). As CT20p-treated MDA-MB-231 cells detached by $6 \mathrm{~h}$ (Figure $5 \mathrm{a}$ ), the early detection of reduced $\beta 1$ integrin was noteworthy. In MDA-MB-231 cells, but not MCF-10A cells, expression of $\alpha 5$ integrin decreased after 3 and $6 \mathrm{~h}$ of CT20p treatment (Figure 5c). The $\alpha \mathrm{V}$ integrin also decreased upon CT20p treatment in MDA-MB-231 cells but not in MCF-10A cells. The decrease in $\alpha 5$ integrin expression detected at $3 \mathrm{~h}$ post CT20p treatment (Figure 5c) mirrored the decreased surface expression of $\beta 1$ seen by flow cytometry (Figure $5 \mathrm{~d}$ ), suggesting that the heterodimeric $\alpha 5 \beta 1$ complex was being affected and could in part be responsible for causing CT20p-mediated cell detachment.

Another consequence of reduced mitochondrial movement induced by CT20p could be decreased actin polymerization. Others have shown that mitochondrial redistribution was 
a

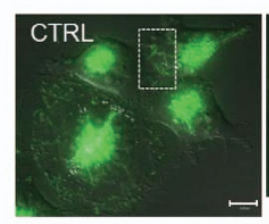

Mitotracker green/DIC

C

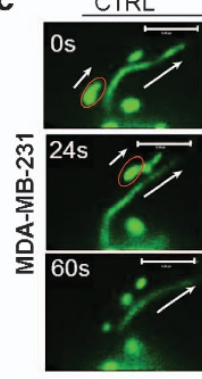

CT20p-2Hr

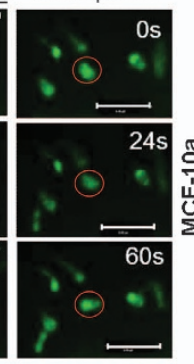

f

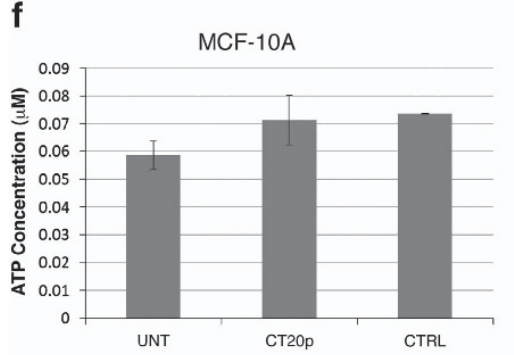

MDA-MB-231
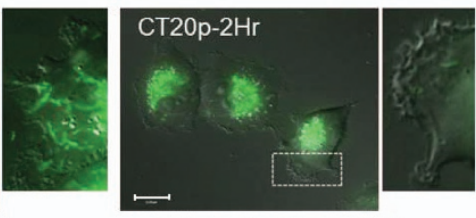

Mitotracker green/DIC b

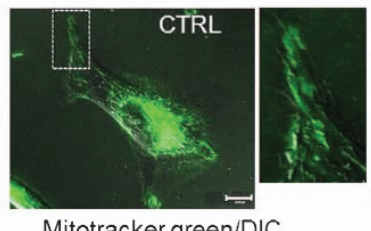

Mitotracker green/DIC
MCF-10A

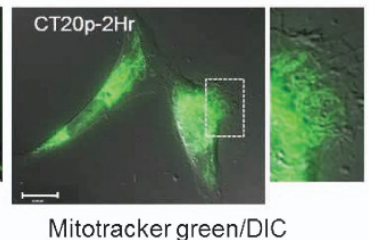

e

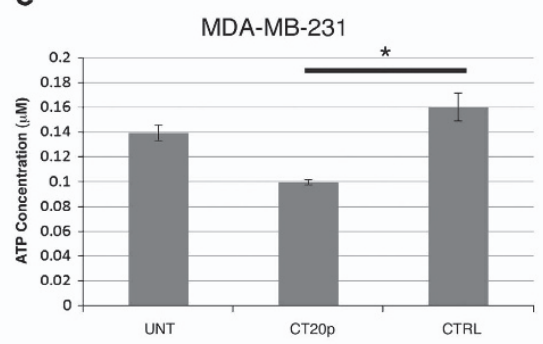

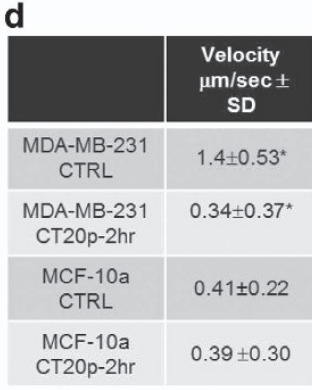

g

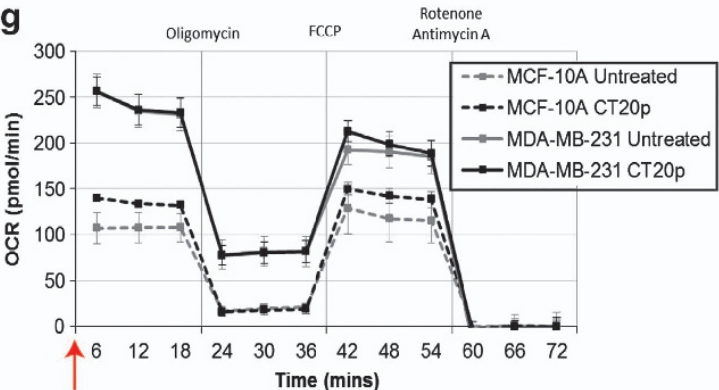

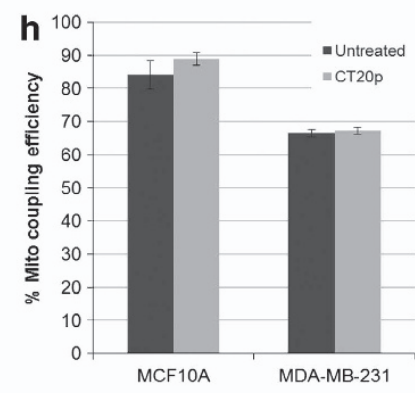

Figure 4 Mitochondrial movement and velocity decreases with CT20p treatment in breast cancer cells. (a, b) MDA-MB-231 cells (a) or MCF-10A cells (b) were untreated or treated with CT20p, delivered in nanoparticles as described in Materials and Methods, for 0-2 $\mathrm{h}$ and stained with Mitotracker green. Time-lapse movies were acquired at five images/sec for 2 min. Representative endpoint images from movies are shown. (c) Digitally magnified images show mitochondria from representative images (a, b). Scale for images is $12 \mu \mathrm{M}(\mathbf{a}, \mathbf{b})$ and $6 \mu \mathrm{M}(\mathbf{c})$. (d) Mitochondrial velocity calculations were made with Volocity software (Perkin Elmer) using data from (a, b). (e, f) Intracellular ATP levels were measured in MDA-MB-231 cells (e) and MFC-10A cells (f) untreated (UNT), treated with CT20p as above or an irrelevant peptide (CTRL) for $3 \mathrm{~h}$. ${ }^{*} P>0.05$. $(\mathbf{g}, \mathbf{h})$ MDA-MB-231 or MCF-10A cells were untreated or treated with CT20p as above for $3 \mathrm{~h}$ at which point (see red arrow) mitochondrial stress analysis was performed using the Seahorse XFe24 analyzer. Oxygen consumption (g) and mitochondrial coupling efficiency (h) were determined following timed additions of inhibitors as described in Materials and Methods. Assay time was $\sim 2 \mathrm{~h}$, for a total CT20p treatment time of $\sim 5 \mathrm{~h}$

required for actin polymerization. ${ }^{6}$ To examine this, cells were probed with Mitotracker Red, the F-actin-specific dye phalloidin-AF633 (pseudo-colored green), the nuclear stain DAPI (blue) and imaged by confocal microscopy. CT20p treatment for 3 and $6 \mathrm{~h}$ in MDA-MB-231 cells impaired mitochondrial distribution and dramatically reduced the amount of F-actin detected (Figure 6a). In control MDA-MB-231 cells (0h), as well as control and CT20p-treated MCF-10A cells, F-actin was detectable (Figures $6 a$ and b). F-actin levels were quantitated and they revealed a general decrease in $F$-actin in CT20p-treated MDA-MB-231 cells over time, with statistically significant changes noted at the 6 -h time point compared with control cells (Figure 6c). No significant changes were noted in the expression of beta-actin in either cell type (Figure 6d). Therefore, we concluded that CT20p, in addition to its actions on the mitochondria, inhibited the polymerization of actin filaments in breast cancer cells but not in normal breast epithelial cells.

CT20p causes breast cancer cell death in vitro and in vivo. To continue our investigation into the cytotoxic effects of CT20p, we examined the lethality of the peptide under hypoxic conditions associated with tumor environments. Increased cell death of MDA-MB-231 cells that were treated with CT20 $\mathrm{p}$ for $48 \mathrm{~h}$ was detected under hypoxiamimicking conditions (Figure 7a), indicating that the cytotoxicity of the peptide was likely unimpaired under low-oxygen conditions.

Next, we assessed the capacity of CT20p to act as a therapeutic agent in vivo in a murine breast tumor model. MDA-MB-231 cells were subcutaneously implanted in mice, and tumor growth was measured. Groups of mice with tumors $\left(\sim 5-8 \mathrm{~mm}^{2}\right)$ were given two sets of intravenous injections of CT20p over a 2-week period. Post-treatment tumor size was monitored by ultrasound (every 2-3 days). Two different HBPE-NPs were used for delivery of CT20p: untargeted carboxylated $(\mathrm{COOH})$ nanoparticles (used in in vitro experiments) and folate-decorated (FOL) nanoparticles that target cells expressing folate receptors, like MDA-MB-231 cells. ${ }^{27}$ From our previous studies, we knew that untargeted and folate-targeted nanoparticles were equally effective in vitro; however, we anticipated that the folate-targeted nanoparticles 

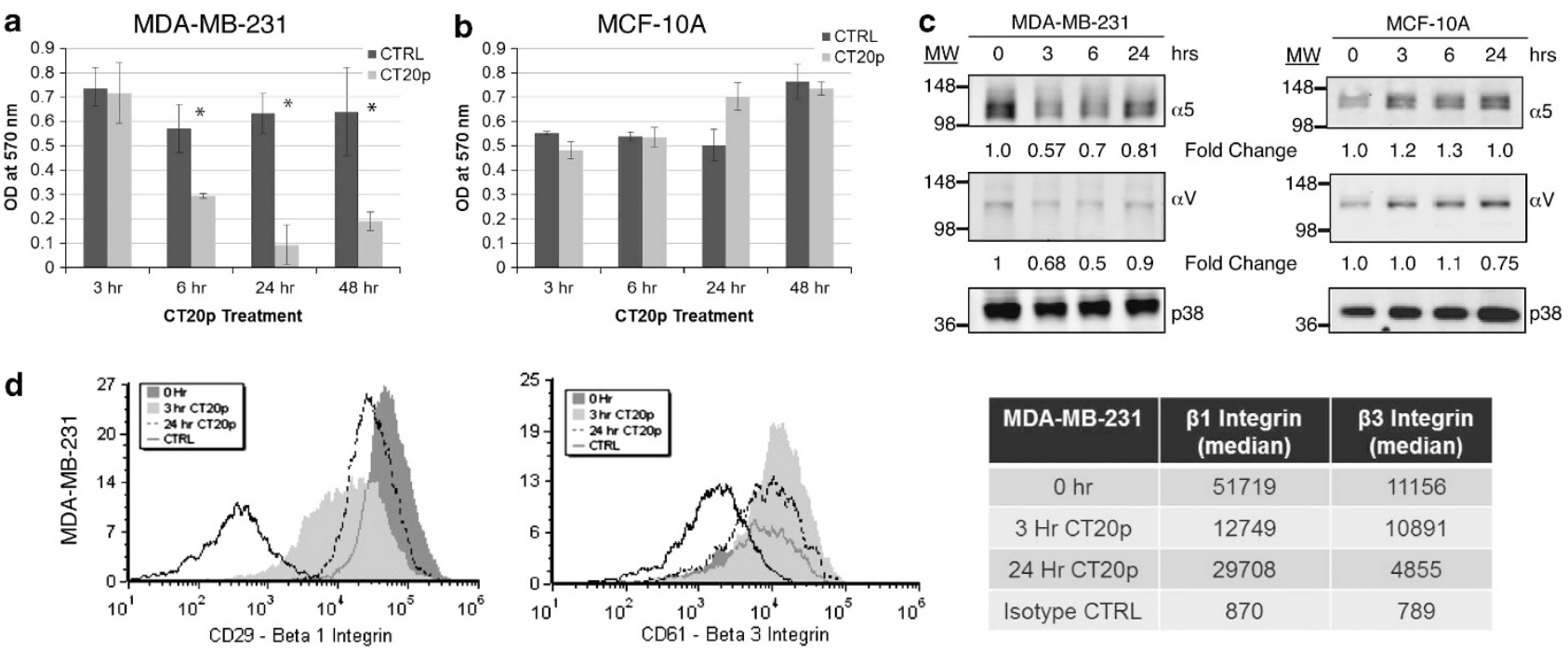

\begin{tabular}{|c|c|c|}
\hline MDA-MB-231 & $\begin{array}{c}\boldsymbol{\beta} \text { 1 Integrin } \\
\text { (median) }\end{array}$ & $\begin{array}{c}\boldsymbol{\beta} \text { 3 Integrin } \\
\text { (median) }\end{array}$ \\
\hline O hr & 51719 & 11156 \\
\hline 3 Hr CT20p & 12749 & 10891 \\
\hline 24 Hr CT20p & 29708 & 4855 \\
\hline Isotype CTRL & 870 & 789 \\
\hline
\end{tabular}
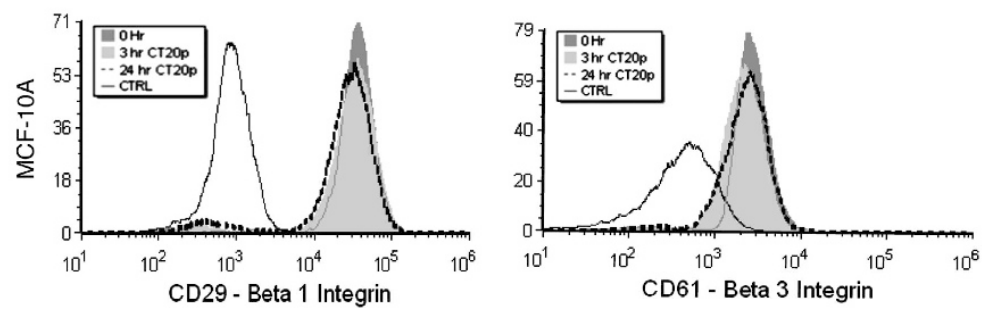

\begin{tabular}{|c|c|c|}
\hline MCF-10A & $\begin{array}{c}\boldsymbol{\beta} \text { 1 Integrin } \\
\text { (median) }\end{array}$ & $\begin{array}{c}\boldsymbol{\beta} \text { Integrin } \\
\text { (median) }\end{array}$ \\
\hline $0 \mathrm{hr}$ & 35321 & 2612 \\
\hline $3 \mathrm{Hr} \mathrm{CT} 20 \mathrm{p}$ & 33482 & 2119 \\
\hline $24 \mathrm{Hr}$ CT20p & 28741 & 2350 \\
\hline Isotype CTRL & 852 & 359 \\
\hline
\end{tabular}

Figure 5 CT20p treatment reduces cell adhesion and integrin levels in breast cancer cells. (a, b) MDA-MB-231 (a) and MCF-10A (b) cells were untreated (CTRL) or treated with CT20p, delivered in nanoparticles as described in Materials and Methods, and cell adhesion measured at time points indicated using a standard crystal violet adhesion assay. ${ }^{*} P<0.05$. (c) Cell lysates from MDA-MB-231 and MCF-10A cells, treated with CT20p as above for $0,3,6$, and $24 \mathrm{~h}$, were immunoblotted for expression of $\alpha 5$ and $\alpha \mathrm{V}$ integrins. p38 MAPK is shown as a loading control for whole-cell lysates. Fold changes refer to the representative blots shown. Images were cropped to improve presentation. (d) MDA-MB-231 and MCF-10A cells, treated with CT20p as above for 0,3 and $24 \mathrm{~h}$, were analyzed for $\beta 1$ (CD29) and $\beta 3$ (CD61) integrins by flow cytometry using fluorescently-tagged antibodies as described in Materials and Methods. Median peak values are shown in table

would concentrate more effectively in tumors when introduced intravenously. Further, to improve circulation and prevent uptake by the reticuloendothelial system, nanoparticles were pegylated, which did not impair uptake by cells as shown using nanoparticles loaded with a dye (Supplementary Figure 4A). Mice were treated twice with HBPE-NPs and the net change in tumor size, determined during a 14-day period for each group of mice, is shown in Figure 7b. When CT20p was delivered in $\mathrm{COOH}-\mathrm{NPs}$, tumor growth was inhibited. When delivered in FOL-NPs, CT20p caused significant tumor regression, with little to no tumor detected after 14 days. In contrast, tumors continued to grow in mice receiving only PBS or HBPE-NPs with an irrelevant peptide. As a positive control, mice received FOL-targeted doxorubicin (DOX). CT20p proved as effective if not better than DOX, an established agent employed in breast cancer chemotherapy. Hematoxylin \& eosin $(H$ \& $E)$ stained sections of the liver and spleen (where untargeted HBPE-NPs could accumulate) from CT20p-treated mice were evaluated by a pathologist and found to have no overt signs of necrosis or damage, whereas targeted tumor tissue did display areas of necrosis (Supplementary Figure 4B). In a representative experiment shown in Figures 7c and d, we display ultrasound images from three mice in which the tumor's growth was monitored for $\sim 1$ month. During this time, one mouse, whose tumor was $\sim 8 \mathrm{~mm}^{2}$ after two weeks, was treated with FOL-CT20p twice, whereas another mouse was treated with FOL-CT20p in a similar manner but the tumor had grown for 1 week and was $\sim 1.5 \mathrm{~mm}^{2}$. In both instances, CT20p impaired tumor growth indicating that its activity could be independent of tumor size, although it should be noted that tumor vascularity can affect intravenous delivery of drugs. Although preliminary, these results indicate that CT20p encapsulated in nanoparticles can be targeted to tumors and as such has promising use as an anticancer agent.

\section{Discussion}

In our study, we describe a novel therapeutic peptide, CT20p, which displays cancer-specific cytotoxic activity. In susceptible cancer cells, CT20p localized to mitochondria and promoted fusion-like aggregation, mitochondrial membrane hyperpolarization, and hindered mitochondrial movement. Furthermore, CT20p reduced integrin expression and impaired polymerization of the actin cytoskeletal prior to cancer cell detachment and death. As a result, treatment of mice bearing subcutaneous tumors with CT20p, delivered in HBPE-NPs targeted to the folate receptor, led to complete tumor regression. Therefore, CT20p, by disrupting mitochondrial redistribution and the cytoskeleton, could limit metastatic cancer cell movement and hasten the death of these cells. 
a

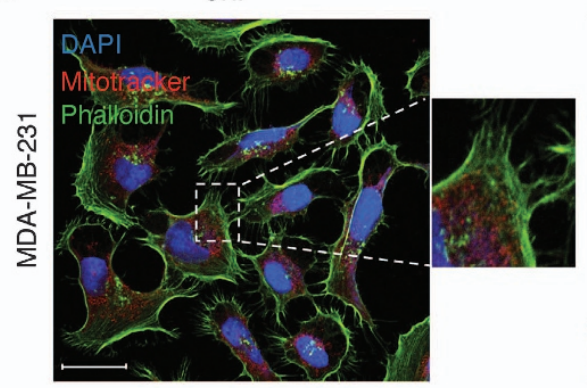

b
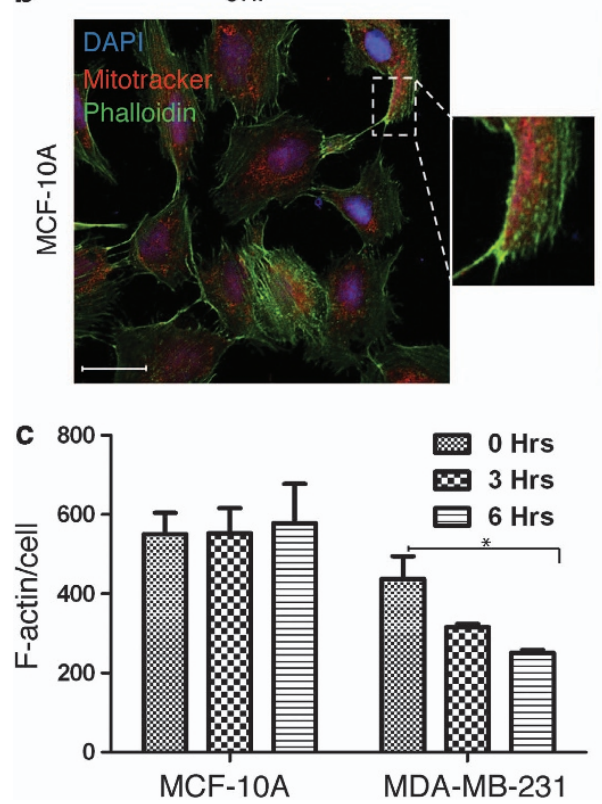

3hr-CT20p

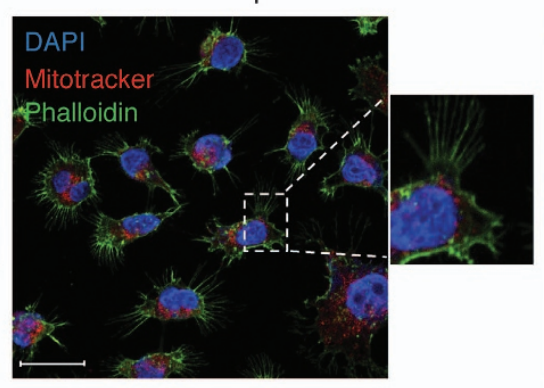

3hr-CT20p

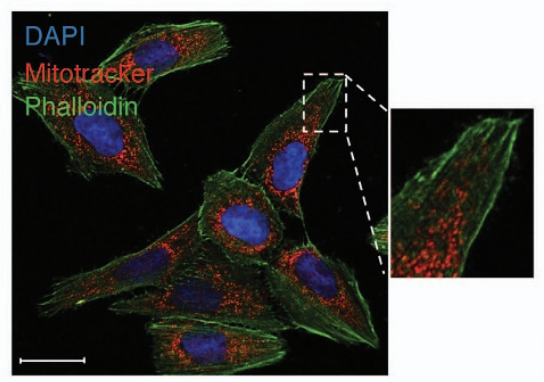

d

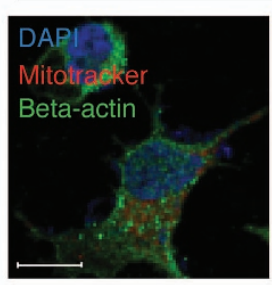

Ohr
MDA-MB-231

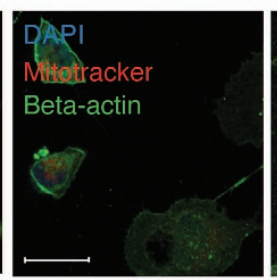

3hr-CT20p

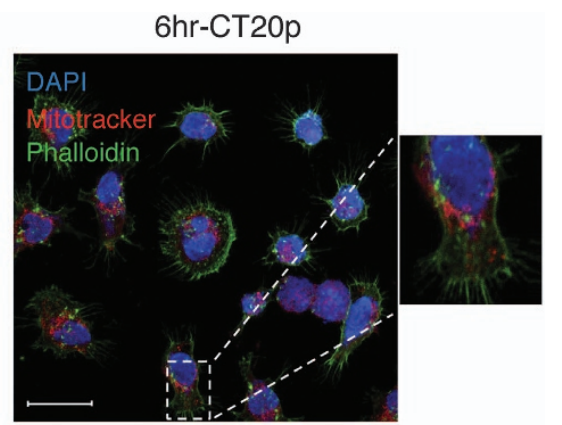

6hr-CT20p

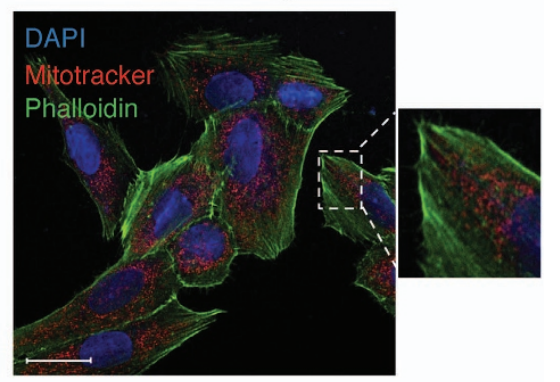

MCF-10A

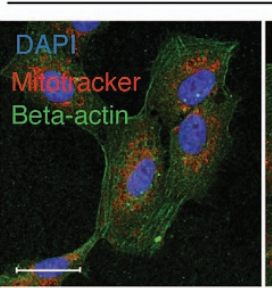

$\mathrm{Ohr}$

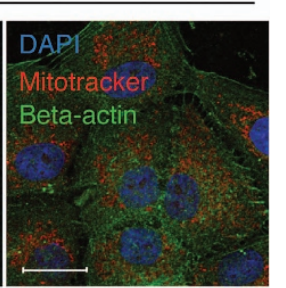

3hr-CT20p

Figure 6 Detection of F-actin is reduced upon CT20p treatment in breast cancer cells. (a, b) MDA-MB-231 cells (a) and MCF-10A cells (b) were treated with CT20p, delivered in nanoparticles as described in Materials and Methods, for the times indicated. Cells were stained with Mitotracker red and then fixed and stained with DAPI (nucleus) (blue) and Phalloidin for F-actin (pseudo-colored green) as described in Materials and Methods. Scale shown is $20000 \mathrm{nM}$ or $20 \mu \mathrm{M}$ and inset magnification scale is $5 \mu \mathrm{M}$. (c) Average F-actin per cell was determined as described in Materials and Methods. ${ }^{*} P<0.05$. (d) Cells above were treated with CT20p for $3 \mathrm{~h}$ and then Mitotracker red was added. Cells were fixed and stained with DAPI (nucleus) (blue) and b-actin (total actin) (green). Images were visualized by fluorescent microscopy. Scale is $20000 \mathrm{~nm}$ or $20 \mu \mathrm{M}$

Mitochondria are highly motile organelles, which possess the capability to re-localize to subcellular regions of a cell depending on local energy demands. ${ }^{4}$ Recent evidence indicates that mitochondria in cancer cells are physiologically different from non-transformed cells. ${ }^{6}$ As example, cancer cell mitochondria preferentially rely on HSP90 chaperones to maintain energy production during tumor progression and metastasis. ${ }^{28}$ Two other studies suggested a role for dynamic mitochondrial changes in the support of cancer cell motility. 5,6 Silencing the expression of the fission protein Drp1 in MDAMB-231 and MDA-MB-436 cells caused mitochondria to assume an elongated morphology and reduced the invasiveness of these cells. ${ }^{6}$ Further, the polarity of mitochondrial localization in metastatic cells was associated with cell directional movement, and the disruption of fusion and fission interfered with mitochondrial localization and cell migration velocity. ${ }^{5}$ Therefore, altering the fragmented mitochondrial phenotype that may be a metabolic adaptation in metastatic breast cancer cells could impair essential biological activities upon which these cells rely. Indeed, deregulation of mitochondrial fission can alter cell cycle progression, impair mitochondrial function, and promote mitochondrial loss. ${ }^{29,30}$

There is precedent for the effect of CT20p upon mitochondrial physiology. A study of the soluble, monomeric form of Bax revealed that it interacts with and regulates MFN2 homotypic complex formation, thus favoring mitochondrial fusion. ${ }^{31}$ This fusion-inducing activity of Bax is separable from the oligomerized form which inserts into the mitochondrial membrane to induce apoptosis. How Bax interacts with MFN2 to regulate fusion and if a particular domain within Bax is driving this interaction remains to be determined. It is possible that the $\mathrm{C}$ terminus of Bax could exhibit similar actions towards MFN2 as does the full-length protein given our findings that CT20p promotes a fusion-like aggregation of mitochondria that is accompanied by elevated levels of MFN2. As an alternative explanation, existing literature suggests that, by forming a pore, Bax could participate or favor mitochondrial fusion. $^{32}$ In previous studies of CT20p, we found that the peptide formed pores in mitochondrial-like lipid vesicles $^{15}$ and, in this manner, enable the fusion or 
a

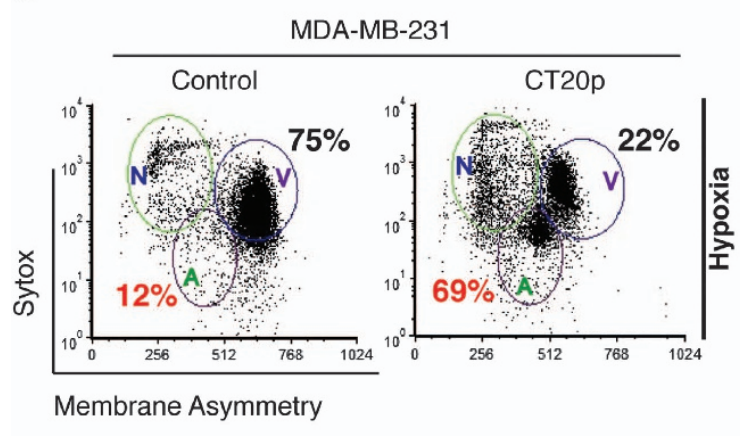

C
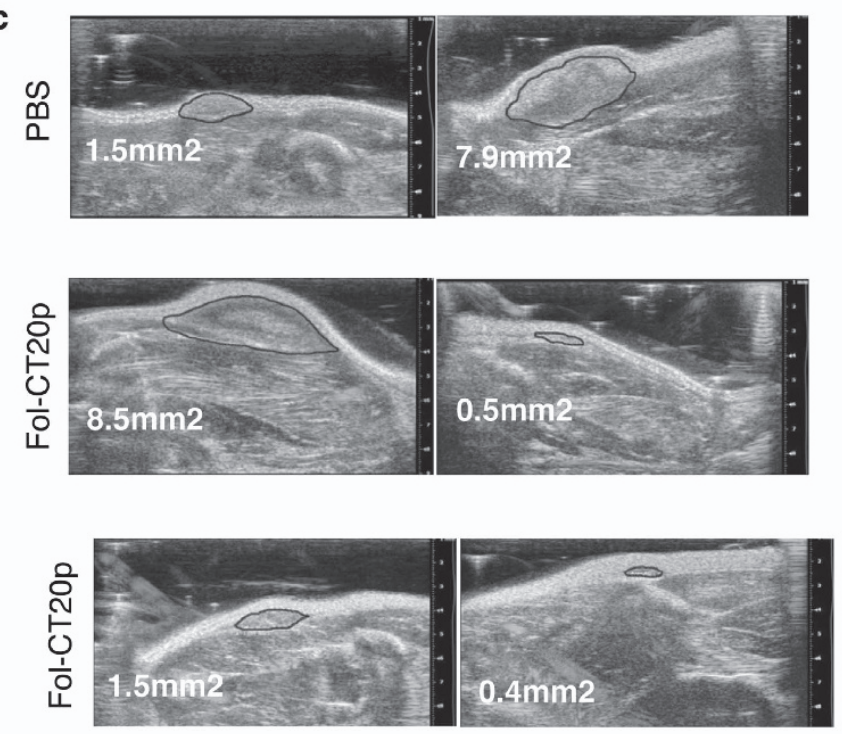
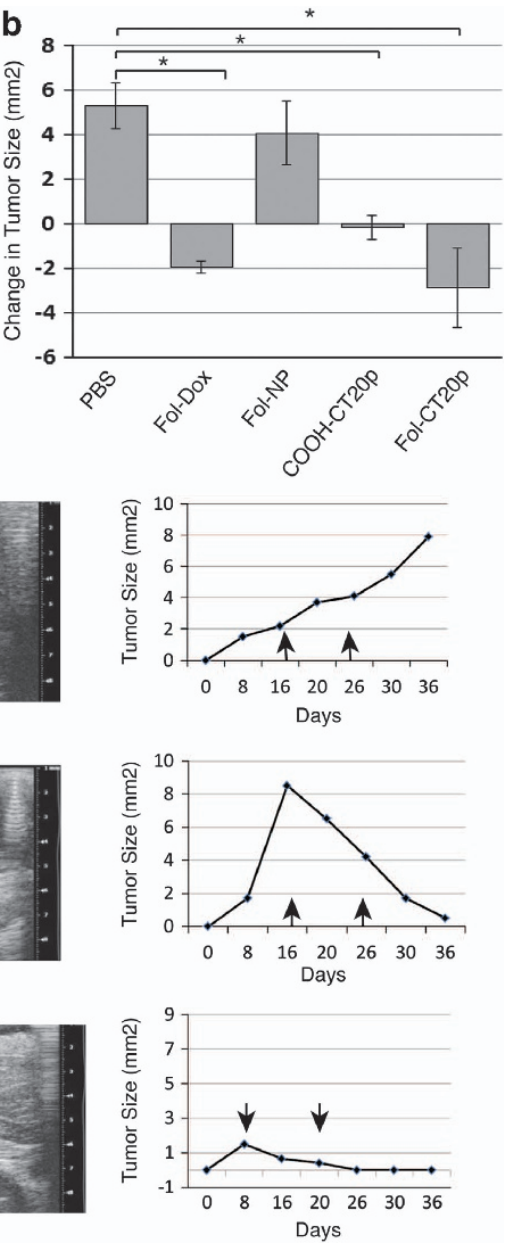

Figure 7 CT20p treatment impairs growth of breast tumors implanted in mice. (a) MDA-MB-231 cells were treated with CT20p under hypoxia-mimicking conditions as described in Materials and Methods. After $48 \mathrm{~h}$, cells were stained with Sytox AADvanced and F2N12S dyes, and cell viability was assessed by flow cytometry (see Figure 1a). Populations are denoted as V (viable), A (apoptotic), and N (necrotic). Percentages of viable cells (black) and apoptotic/necrotic cells (red) are shown. (b) Mice ( $n=5)$ with subcutaneous tumors (MDA-MB-231 cells) were treated twice (after $\sim 0$ and 7 days from tumor detection) with PBS control, folate-receptor targeted doxorubicin (FOL-Dox), folate-receptor targeted control nanoparticles with an irrevelant peptide (FOL-NP) or folate-receptor targeted CT20p nanoparticles (FOL-CT20p) over a 2-week period as described in Materials and Methods. Non-targeted nanoparticles loaded with CT20p (COOH-CT20p) were also used. ${ }^{*} P<0.05$. (c) Representative ultrasound images from mice treated with PBS, or FOL-CT20p acquired at experimental endpoints are shown. Tumor growth curves indicate the size of tumors and the times (arrows) of treatment over a 36-day period

aggregation of mitochondria. CT20p could also interfere with the movement of cellular components on microtubules and retard the transport of cargo, like mitochondria or integrins.

The effective use of therapeutic peptides derived from endogenous proteins is challenged by the lack of optimal delivery strategies. In our studies, we capitalized on the hydrophobic nature of CT20p and the use of HBPE-NPs that can be conjugated to targeting ligands to guide them to specific cell surface targets. ${ }^{16}$ The HBPE-NPs protected CT20p while in circulation and allowed efficient uptake by targeting tumor cells. Once taken up by cells, the peptide was released from the nanoparticles and bound to mitochondria in cancer cells as we have shown in our studies. Further, we validated that CT20p affects the cytoskeleton, a 'druggable node' for metastatic disease ${ }^{33}$ in part by impairing mitochondrial redistribution to energy-demanding regions of the cell, like cell protrusions. The next step to fully exploit the clinical utility of CT20p, and is the focus of continuing studies, is identifying the unique aspects of cancer cell mitochondria that are targeted by the peptide.

\section{Materials and Methods}

Cell culture and reagents. MDA-MB-231 cells (ATCC HTB-26, Manassas, VA, USA) were cultured in DMEM (Cellgro, Manassas, VA, USA) with $10 \%$ fetal bovine serum and $1 \%$ penicillin-streptomycin. MCF-10A cells (ATCC CRL-10317) were cultured in mammary epithelial cell growth media (Lonza, Walkersville, MD, USA) with $1 \%$ penicillin-streptomycin. Cells were authenticated by STR profiling (ATCC). CT20p (Ac-VTIFVAGVLTASLTIWKKMG-NH2) and RHO-tagged CT20p were commercially synthesized (Biopeptide Co., Inc, San Diego, CA, USA) at $>98 \%$ purity. Human tissues were transported in RPMI on ice. Upon receipt, tissues were washed thoroughly in PBS containing $1 \%$ penicillin-streptomycin and $5 \mu \mathrm{g} / \mathrm{ml}$ Fungizone (Life Technologies, Carlsbad, CA, USA). Tissues were minced and digested in $0.1 \%$ collagenase I (Gibco, Carlsbad, CA, USA) at $37^{\circ} \mathrm{C}$ for $2 \mathrm{~h}$. Differential centrifugation as described by Speirs et al. ${ }^{34}$ was used to obtain an epithelial-enriched cell fraction. Cells were maintained in mammary epithelial cell growth media (Lonza) at $37^{\circ} \mathrm{C}$ and $5 \% \mathrm{CO}_{2}$. Human tissues were deidentified and the protocol for use was approved by the Institutional Review Boards at the University of Central Florida and Florida Hospital. 
CT20p-nanoparticle synthesis. CT20p was encapsulated into HBPE-NPS following a previously reported method. ${ }^{11,16}$ In brief, $36 \mu$ l of CT20p $(0.05 \mu \mathrm{g} / \mu \mathrm{l})$ solution in $250 \mu \mathrm{l}$ of DMSO were mixed in $250 \mu \mathrm{l}$ of a DMSO solution containing the HBPE polymer $(12 \mathrm{mg})$ for a ratio of $\sim 0.15 \mu \mathrm{g}$ peptide: $1 \mathrm{mg}$ nanoparticles. The resulting polymer/CT20p mixture in DMSO was added to deionized water $(2.5 \mathrm{ml})$ to form the HBPE (CT2Op) NPs. The resulting NPs were purified using a PD-10 column and dialyzed (MWCO 6-8K) against PBS $(\mathrm{pH}=7.4)$. Dynamic light scattering and zeta potential analysis of the nanoparticle reveals a size diameter of $88 \pm 2 \mathrm{~nm}$ and zeta potential of $-54.5 \mathrm{mV}$. The HBPE-NPs (above) contain functional carboxylic groups on their surface that results in a negative charge. For targeting the folate receptor to concentrate the nanoparticles in murine tumors, folic acid was conjugated to HBPE-NPs and nanoparticles pegylated as previously described. $^{35}$

Assays of cell viability. Short-term survival was assessed using the flow cytometry based Sytox AADvanced and F2N12S Violet Ratiometric Apoptosis kit according to the manufacturer's protocol (Invitrogen, Carlsbad, CA, USA). Longterm survival of cells was measured by clonogenic survival assay. ${ }^{36}$ The dose of CT20p that kills $\sim 50 \%$ of the cells in $48 \mathrm{~h}$ was determined to be $\sim 3.5 \mathrm{nM}(75 \mu \mathrm{g}$ nanoparticles $/ \mathrm{ml}$ ) (Supplementary Figure 1). To induce hypoxia-mimicking conditions, $200 \mu \mathrm{M} \mathrm{CoCl}_{2}$ was added to the media.

Live cell imaging. Cells were plated in $35-\mathrm{mm}$ glass-bottom dishes (MatTek, Ashland, MA, USA) coated with $10 \mu \mathrm{g} / \mathrm{ml}$ fibronectin (Sigma, Milwaukee, WI, USA) for MDA-MB-231 or $3.25 \mu \mathrm{g} / \mathrm{cm}^{2}$ Cell-Tak (BD Biosciences, San Diego, CA, USA) for MCF-10A cells. Cells were treated with CT20p and stained with $25 \mathrm{nM}$ Mitotracker Green or $500 \mathrm{nM}$ ER-Tracker Green (Life Technologies). Images were acquired with a PerkinElmer UltraView spinning disc confocal system, with AxioObserver.Z1 stand (Carl Zeiss, Thornwood, NY, USA), in a humidity and temperature-controlled chamber (LiveCell, Seoul, Korea). Time-lapse movies were acquired using a PlanApochromat $63 \times$ Oil DIC objective. Images were processed with Volocity software (PerkinElmer, Shelton, CT, USA). Live cell imaging was also used to track mitochondrial movement and velocities calculated using the Volocity software (PerkinElmer).

Detection of mitochondrial fusion. Cells, seeded in 24-well glassbottom dishes (MatTek), were transfected with mitoDendra ${ }^{17}$ using TransIT-LT1 transfection reagent (Mirus, Madison, WI, USA) according to the manufacturer's protocol and treated with CT20p. Live cell confocal imaging was performed using Zeiss LSM 710 confocal microscope and ZEN 2010 software. Images were obtained using a Zeiss $63 \times$ Plan-Apocromat oil immersion lens (numerical aperture $(N A=1.4)$ ), pinholes adjusted for an optical section thickness of $1 \mu \mathrm{m}$ for each channel, and a $488 / 543$ dual dichroic. Photoconversion of mitoDendra was achieved by exposing a region of interest within a cell to the $488 \mathrm{~nm}$ light from an Argon laser set to $5 \mathrm{~mW}$ (20\% power) with 5-20 iterations (depending on the mitoDendra expression levels) at a pixel dwell time of $1.27 \mu \mathrm{s}$. Nonphotoconverted mitoDendra was visualized by excitation at $488 \mathrm{~nm}$ (Argon laser at $0.05 \mathrm{~mW}, 0.2 \%$ power) and emission light collected at $500-540 \mathrm{~nm}$. Photoconverted mitoDendra was visualized by excitation at $543 \mathrm{~nm}$ (HeNe543 laser at $0.13 \mathrm{~mW}, 10 \%$ power) and emission light collected at $555-700 \mathrm{~nm}$. Fluorescence for the non- and photoconverted mitoDendra were captured separately, whereas DIC images were captured simultaneously. Maximum intensity projections were created and the resulting single z-slice movies were optimized using ZEN 2010 software. MFN2 overexpression was performed using pMFN-2-YFP (Addgene, Cambridge, MA, USA) and cells transfected as described above.

Measurement of mitochondrial bioenergetics. Culture plates for use in the Seahorse XFe24 analyzer were coated with Cell-Tak (BD Bioscience) at $3.5 \mu \mathrm{g} / \mathrm{cm}^{2}$ and cells seeded at 60000 cells/well. Oxygen consumption rates were measured following the manufacturer's protocol. For metabolic profiling, injections of oligomycin $(1 \mu \mathrm{M}), \operatorname{FCCP}(0.3 \mu \mathrm{M})$, rotenone $(0.1 \mu \mathrm{M})$, and antimycin $\mathrm{A}(2 \mu \mathrm{M})$ were used. The mitochondrial coupling efficiency was calculated as: \% coupling efficiency $=(1-$ (minimum oligomycin response/final basal measurement $)) \times 100$. To measure ATP, cells were treated with CT20p and total cellular ATP determined using the ATPlite luminescence-based kit (PerkinElmer) according to the manufacturer's protocol. Luminescence was read with an Envision plate reader (Perkin Elmer).
Detection of actin polymerization. Immunohistochemistry was performed as previously described. ${ }^{36,37}$ For detection of total actin levels, cells were incubated with a beta-actin primary antibody (Cell Signaling, Danvers, MA, USA) followed by incubation with an AlexaFluor546 conjugated goat anti-mouse secondary antibody (Invitrogen). For detection of F-actin and DNA, cells were stained with AlexaFluorH633-phalloidin and DAPI (Invitrogen). Cells were visualized with a Zeiss LSM 710 microscope. Images shown in figures were obtained using a Zeiss $63 \times$ Plan-Apocromat oil immersion lens, whereas images acquired for F-actin quantification were obtained using a Zeiss $40 \times$ Plan-Apocromat oil immersion lens. To determine the average F-actin levels per cell, the number of pixels above background intensity was quantitated and then divided by the number of cells in the image (Volocity software, Perkin Elmer). At least three images, containing 9-29 cells, per condition were quantified.

Measurement of mitochondrial membrane potential. The mitochondrial membrane potential $(\Delta \psi)$ was measured by flow cytometry $(488 \mathrm{~nm}$ excitation and $530 \mathrm{~nm}$ (green)/585 nm (red) emission) using the dye JC-1 at a final concentration of $1 \mu \mathrm{M}$ (Life Technologies). For live cell imaging, cells were seeded on glass, treated with CT20p, and stained with $1 \mathrm{UM} \mathrm{JC}-1$, and imaged on the Ultraview spinning disk confocal microscope (PerkinElmer) as described above.

Detection of proteins by western blot. Mitochondrial fractionation was performed as previously described. ${ }^{38}$ Mitochondrial lysates or whole-cell lysates were subjected to SDS-PAGE, transferred to Immobilon-FL membranes (Millipore, Temecula, CA, USA) or PVDF membranes and probed with primary antibodies against MFN2 (Millipore), OPA1 (Novus Biologicals, Saint Charles, MO, USA), integrin $\alpha 5$ (Cell Signaling), integrin $\alpha \mathrm{V}$ (Cell Signaling), and prohibitin (Abcam, Cambridge, MA, USA) or p38 MAPK (Santa Cruz, Dallas, TX, USA). Fluorescent detection was achieved by incubation with an IRDye $800 \mathrm{CW}$ anti-mouse or anti-rabbit secondary antibody, followed by imaging with an Odyssey detection system (LI-COR). Densitometry was analyzed using ImageJ software.

Cellular adhesion assay. A standard crystal violet adhesion assay was performed as previously described. ${ }^{39}$ Plates were uncoated or coated with $20 \mu \mathrm{g} / \mathrm{ml}$ fibronectin (Sigma), and cells were seeded at a density of 25000 cells/well. Following treatment with peptides at time points indicated in the figure, cells were fixed and stained with $5 \mathrm{mg} / \mathrm{ml}$ crystal violet. Absorbance at $595 \mathrm{~nm}$ was read on an EnVision plate reader (Perkin Elmer).

Measurement of cell surface integrin expression. Cells, treated with CT20p, were washed with 5\% FBS in PBS and stained with FITC mouse antihuman CD61, PE mouse anti-human CD29 (BD Bioscience), or a corresponding isotype control antibody (BD Bioscience). Data were acquired with an Accuri C6 flow cytometer and analyzed with FCS Express (Denovo, Niwot, CO, USA) software.

In vivo studies. Female, 6-8-week-old, Foxn1nu/Foxn1nu nude mice (Charles River, Troy, NY, USA) received subcutaneous injections of $\sim 10^{6}$ MDA-MB-231 cells. Tumors were detected by ultrasound (VisualSonics Vevo 2100 , Toronto, ON, Canada). Mice bearing tumors $\left(\sim 1.5-8 \mathrm{~mm}^{2}\right)$ received tail vain injections of $5 \mu \mathrm{g}$ HBPE-NPs (untargeted or folate-receptor targeted) encapsulating СТ20p, or control nanoparticles as described in the figure. Folatetargeted doxorubicin was used as a positive control. Two injections, after 0 and 7 days, were administered to each mouse in each group over a 2-week period, and tumor size was monitored by ultrasound. For tissue staining, a standard hematoxylin \& eosin protocol was performed. An animal study protocol was approved by the Institutional Animal Care and Use Committee at the University of Central Florida.

Statistical analysis. For each figure, representative experiments are shown that were replicated a minimum of three times. For microscopy, multiple fields were acquired for each representative image. Two-way ANOVA was used to compare different agents and different time points within each experiment with a statistically significant difference defined as a P-value of $<0.05$. Calculations were performed with Prism (GraphPad, La Jolla, CA, USA). For the mouse studies, given the size of the S.D. of the tumors and the difference in the means between groups of control and treated mice, at a minimum of $n=5$ for each group, at $95 \%$ power the $P$-values were $<0.05$. 


\section{Conflict of Interest}

The authors declare no conflict of interest.

Acknowledgements. We appreciate Dr. Jordi Magrane, Weill Medical College of Cornell University, New York, NY, USA, for providing the mitoDendra vector and Dr. SA Litherland and Dr. David Decker from Florida Hospital, Orlando, FL, USA for the acquisition of patient tissues. This work was supported by grants from NIGMS (GM083324) and the Florida Breast Cancer Foundation to AK.

1. Constance JE, Lim CS. Targeting malignant mitochondria with therapeutic peptides. Ther Deliv 2012; 3: 961-979.

2. Smith RA, Hartley RC, Cochemé HM, Murphy MP. Mitochondrial pharmacology. Trends Pharmacol Sci 2012; 33: 341-352.

3. Szewczyk A, Wojtczak L. Mitochondria as a pharmacological target. Pharmacol Rev 2002 54: 101-127.

4. Campello $\mathrm{S}$ et al. Orchestration of lymphocyte chemotaxis by mitochondrial dynamics J Exp Med 2006; 203: 2879-2886.

5. Desai SP, Bhatia SN, Toner M, Irimia D. Mitochondrial localization and the persistent migration of epithelial cancer cells. Biophys J 2013; 104: 2077-2088.

6. Zhao J, Zhang J, Yu M, Xie Y, Huang Y, Wolff DW et al. Mitochondrial dynamics regulates migration and invasion of breast cancer cells. Oncogene 2013; 32 4814-4824.

7. Boohaker RJ, Lee MW, Vishnubhotla P, Perez JM, Khaled AR. The use of therapeutic peptides to target and to kill cancer cells. Curr Med Chem 2012; 19: 3794-3804.

8. Lien S, Lowman HB. Therapeutic peptides. Trends Biotechnol 2003; 21: 556-562.

9. Kolluri SK, Zhu X, Zhou X, Lin B, Chen Y, Sun K et al. A short Nur77-derived peptide converts Bcl-2 from a protector to a killer. Cancer Cell 2008; 14: 285-298.

10. Arbel N, Shoshan-Barmatz V. Voltage-dependent anion channel 1-based peptides interact with Bcl-2 to prevent antiapoptotic activity. J Biol Chem 2010; 285 6053-6062

11. Boohaker RJ, Zhang G, Lee MW, Nemec KN, Santra S, Perez JM et al. Rational development of a cytotoxic peptide to trigger cell death. Mol Pharm 2012; 9: 2080-2093.

12. Garcia-Saez AJ, Coraiola M, Dalla Serra M, Mingarro I, Menestrina G, Salgado J. Peptides derived from apoptotic Bax and Bid reproduce the poration activity of the parent full-length proteins. Biophys J 2005; 88: 3976-3990.

13. Valero JG, Sancey L, Kucharczak J, Guillemin Y, Gimenez D, Prudent J et al. Bax-derived membrane-active peptides act as potent and direct inducers of apoptosis in cancer cells. J Cell Sci 2011; 124(Pt 4): 556-564.

14. Garg P, Nemec KN, Khaled AR, Tatulian SA. Transmembrane pore formation by the carboxyl terminus of Bax protein. Biochim Biophys Acta 2013; 1828: 732-742.

15. Tatulian SA, Garg P, Nemec KN, Chen B, Khaled AR. Molecular basis for membrane pore formation by Bax protein carboxyl terminus. Biochemistry 2012; 51: 9406-9419.

16. Santra S, Kaittanis C, Perez JM. Aliphatic hyperbranched polyester: a new building block in the construction of multifunctional nanoparticles and nanocomposites. Langmuir 2010; 26 5364-5373.

17. Magrane J, Sahawneh MA, Przedborski S, Estévez ÁG, Manfredi G. Mitochondria dynamics and bioenergetic dysfunction is associated with synaptic alterations in mutan SOD1 motor neurons. J Neurosci 2012; 32: 229-242.

18. Santel A, Fuller MT. Control of mitochondrial morphology by a human mitofusin. J Cell Sci 2001; 114(Pt 5): 867-874

19. Eura Y, Ishihara N, Yokota S, Mihara K. Two mitofusin proteins, mammalian homologues of FZO, with distinct functions are both required for mitochondrial fusion. J Biochem 2003 134: 333-344.

20. Nagae M, Re S, Mihara E, Nogi T, Sugita Y, Takagi J. Crystal structure of alpha5beta1 integrin ectodomain: atomic details of the fibronectin receptor. J Cell Biol 2012; 197 $131-140$

21. Huang $P, Y u T$, Yoon $Y$. Mitochondrial clustering induced by overexpression of the mitochondrial fusion protein Mfn2 causes mitochondrial dysfunction and cell death Eur J Cell Biol 2007; 86: 289-302.
22. Cassidy-Stone A, Chipuk JE, Ingerman E, Song C, Yoo C, Kuwana T et al. Chemical inhibition of the mitochondrial division dynamin reveals its role in Bax/Bak-dependent mitochondrial outer membrane permeabilization. Dev Cell 2008; 14: 193-204.

23. Arismendi-Morillo G, Hoa NT, Ge L, Jadus MR. Mitochondrial network in glioma's invadopodia displays an activated state both in situ and in vitro: potential functional implications. Ultrastruct Pathol 2012; 36: 409-414.

24. Barkan D, Chambers AF. beta1-integrin: a potential therapeutic target in the battle against cancer recurrence. Clin Cancer Res 2011; 17: 7219-7223.

25. Berry MG, Gui GP, Wells CA, Carpenter R. Integrin expression and survival in human breast cancer. Eur J Surg Oncol 2004; 30: 484-489.

26. Desgrosellier JS, Cheresh DA. Integrins in cancer: biological implications and therapeutic opportunities. Nat Rev Cancer 2010; 10: 9-22.

27. Meier R, Henning TD, Boddington S, Tavri S, Arora S, Piontek G et al. Breast cancers: MR imaging of folate-receptor expression with the folate-specific nanoparticle P1133. Radiology 2010; 255: 527-535.

28. Caino MC, Chae YC, Vaira V, Ferrero S, Nosotti M, Martin NM et al. Metabolic stress regulates cytoskeletal dynamics and metastasis of cancer cells. J Clin Invest 2013; 123: 2907-2920.

29. Parone PA, Da Cruz S, Tondera D, Mattenberger $Y$, James DI, Maechler P et al. Preventing mitochondrial fission impairs mitochondrial function and leads to loss of mitochondrial DNA. PLoS One 2008; 3: e3257.

30. Rehman J, Zhang HJ, Toth PT, Zhang Y, Marsboom G, Hong Z et al. Inhibition of mitochondrial fission prevents cell cycle progression in lung cancer. FASEB J 2012; 26: 2175-2186.

31. Hoppins S, Edlich F, Cleland MM, Banerjee S, McCaffery JM, Youle RJ et al. The soluble form of Bax regulates mitochondrial fusion via MFN2 homotypic complexes. Mol Cell 2011; 41: $150-160$

32. Papanicolaou KN, Phillippo MM, Walsh K. Mitofusins and the mitochondrial permeability transition: the potential downside of mitochondrial fusion. Am J Physiol Heart Circ Physiol 2012; 303: H243-H255

33. Quintela-Fandino M, Gonzalez-Martin A, Colomer R. Targeting cytoskeleton reorganisation as antimetastatic treatment. Clin Transl Oncol 2010; 12: 662-669.

34. Speirs V, Green AR, Walton DS, Kerin MJ, Fox JN, Carleton PJ et al. Short-term primary culture of epithelial cells derived from human breast tumours. $\mathrm{Br} J$ Cancer 1998; 78 : 1421-1429.

35. Santra S, Perez JM. Selective N-alkylation of beta-alanine facilitates the synthesis of a poly(amino acid)-based theranostic nanoagent. Biomacromolecules 2011; 12: 3917-3927.

36. Lee MW, Kim WJ, Beardsley DI, Brown KD. N-methyl-N'-nitro-N-nitrosoguanidine activates multiple cell death mechanisms in human fibroblasts. DNA Cell Biol 2007; 26, pp 683-694.

37. Sparrow N, Manetti ME, Bott M, Fabianac T, Petrilli A, Bates ML et al. The actin-severing protein cofilin is downstream of neuregulin signaling and is essential for Schwann cell myelination. J Neurosci 2012; 32: 5284-5297.

38. Yamaguchi $\mathrm{H}$, Paranawithana SR, Lee MW, Huang Z, Bhalla KN, Wang HG. Epothilone B analogue (BMS-247550)-mediated cytotoxicity through induction of Bax conformational change in human breast cancer cells. Cancer Res 2002; 62: 466-471.

39. Humphries MJ. Cell adhesion assays. Mol Biotechnol 2001; 18: 57-61.

(2) () $\odot$ Cell Death and Disease is an open-access journal BY ${ }_{\mathrm{BC}}$ published by Nature Publishing Group. This work is licensed under a Creative Commons Attribution-NonCommercialNoDerivs 3.0 Unported License. The images or other third party material in this article are included in the article's Creative Commons license, unless indicated otherwise in the credit line; if the material is not included under the Creative Commons license, users will need to obtain permission from the license holder to reproduce the material. To view a copy of this license, visit http://creativecommons.org/ licenses/by-nc-nd/3.0/

Supplementary Information accompanies this paper on Cell Death and Disease website (http://www.nature.com/cddis) 